\title{
Grands et petits planteurs de caoutchouc en Afrique (1934-1973)
}

Large and small rubber producers in Africa, 1934 to c. 1973

William G. Clarence-Smith

\section{(2) OpenEdition}

Édition électronique

URL : http://journals.openedition.org/economierurale/3506

DOI : 10.4000/economierurale.3506

ISSN : 2105-2581

Éditeur

Société Française d'Économie Rurale (SFER)

Édition imprimée

Date de publication : 30 juillet 2012

Pagination : 88-102

ISSN : 0013-0559

Référence électronique

William G. Clarence-Smith, « Grands et petits planteurs de caoutchouc en Afrique (1934-1973)»,

Économie rurale [En ligne], 330-331 I juillet-septembre 2012, mis en ligne le 30 juillet 2014, consulté le 19 avril 2019. URL : http://journals.openedition.org/economierurale/3506 ; DOI : 10.4000/ economierurale.3506

(c) Tous droits réservés 


\section{Grands et petits planteurs de caoutchouc en Afrique (1934-1973)}

William G. CLARENCE-SMITH • University of London, School of Oriental and African Studies, SOAS, Londres

\section{Introduction}

A u début des années 1930, le caoutchouc africain ne semblait avoir aucun avenir. En 1932, le prix tombait à son niveau le plus bas, en termes réels. (Barlow et Drabble 1990). Une année plus tard, l'Afrique ne comptait plus que pour $0,2 \%$ de la production mondiale, comparé à 21,2\% en 1910 (Hailey 1938 ; Drabble, 1973). Mais la conjoncture internationale belliqueuse, se terminant par la conquête japonaise du SudEst asiatique en 1941-1942, a redonné de la vigueur au secteur. Après 1945, la longévité de l'hévéa, ainsi que la guerre froide et les troubles politiques en Asie ont soutenu la production en Afrique pendant un certain temps. La perte de l'Indochine, en 1954, a mené certaines grandes sociétés à chercher de nouveaux terrains en Afrique française.

Les indépendances africaines ont miné ce progrès dans beaucoup de pays, au moment même où l'Asie sortait de ses crises. Même les pays africains qui ont le mieux géré leur liberté, par exemple la Côte d'Ivoire, ont misé sur les grandes plantations, quand les petits planteurs triomphaient en Asie. La flambée des prix du pétrole en 1973 a freiné l'ascension du caoutchouc synthétique en Occident, tout en relançant le caoutchouc naturel en Asie, mais l'Afrique s'est trouvée en mauvaise posture pour profiter de cette nouvelle conjoncture.

Au départ, le renouvellement du caoutchouc semblait passer par l'implantation de grandes compagnies occidentales, qui maîtrisaient les techniques mises en place en Asie, et qui bénéficiaient de capitaux considérables. Et les petits planteurs ayant adopté ces techniques, du moins celles qui leur semblaient efficaces, disposèrent d'atouts formidables. Ils ne payaient généralement rien pour leurs terres, connaissaient mieux les conditions locales que les Européens, et se contentaient des outils qu'ils possédaient déjà. Surtout, ils disposaient d'une main-d'œuvre peu chère, soit familiale, soit immigrée. Leurs coûts de production étaient donc très largement inférieurs à ceux des grandes compagnies, même si la qualité de leur caoutchouc était souvent moins bonne. Cependant, d'une manière générale, l'Afrique n'a pas connu le succès des petits planteurs indigènes, qui caractérisait l'Indonésie, la Malaisie et la Thaïlande. L'exception notoire était le Nigéria, mais des politiques agricoles désastreuses après l'indépendance ont étouffé ce paysannat précoce du caoutchouc.

\section{4-1939 \\ Les grandes plantations et les transferts de technologie}

L'International Rubber Regulations Agreement (IRRA), signé en 1934, a relancé la production africaine de caoutchouc. L'IRRA ne concernait que l'Asie, permettant aux planteurs en Afrique de bénéficier d'une lente remontée des prix, sans qu'ils soient astreints aux contraintes à la production. Aussi, la mécanisation rapide des forces armées multipliait la production de pneus et d'autres objets en caoutchouc, dans une conjoncture politique menaçante après l'invasion japonaise de la Manchourie en 1931 (McFadyean, 1944).

La plus grande firme à se lancer dans le caoutchouc africain était la Firestone Tire and Rubber Company, un des quatre géants du pneu américain. Dès le Plan Stevenson de 1922, la Firestone avait cherché à déve- 
lopper des plantations en dehors des possessions britanniques et néerlandaises. Le champignon Microcyclus ulei ravageant l'Amérique latine, et la législation foncière faisant obstacle aux Philippines, le choix s'était porté sur le Libéria en 1924 . Ce pays africain était indépendant, mais sous forte influence américaine. La signature de l'IRRA en 1934 a relancé l'affaire, et la compagnie a saigné ses premiers hévéas en cette année.

Firestone a employé des agronomes néerlandais, et a investi dans la technique de la greffe de boutures, amenant du matériel végétal d'Indonésie, et formant la maind'œuvre locale. En 1940, les trois-quarts des hévéas étaient des clones à haut rendement, plantés sur presque 30000 hectares. En outre, la moitié de la production (autour de 7000 tonnes) était exportée sous forme de latex liquide, une technologie de pointe (Lief, 1951). Les chefs africains, soutenus par les forces armées, fournissaient des travailleurs « libres », contre paiement par le Gouvernement (Kraaij, 1983 ; Salles, 1979).

La Société financière des caoutchoucs (Socfin) représentait un tout autre type de compagnie. Avec son siège à Bruxelles, mais dominée par des capitaux français, la Socfin était le principal producteur de caoutchouc en Indochine dans les années 1920, et disposait de vastes plantations en Indonésie et en Malaisie. En revanche, ses domaines en Afrique produisaient surtout de l'huile de palme et du café (Clarence-Smith, 1997 ; Clarence-Smith, 2010).

À partir de 1926, la Socfin s'est tournée vers le Cameroun sous tutelle française pour planter des hévéas. Une filiale disposait d'une ancienne concession allemande à Dizangué, près de Douala, contenant déjà un peu de caoutchouc ${ }^{1}$. Les primes à l'exportation versées par le gouvernement de 1931 à 1935, ainsi que le régime de travail forcé, ont encouragé l'hévéaculture pendant la crise économique (Etoga Eily, 1971). En

\footnotetext{
1. Rapports annuels de la Socfin.
}

1938, quand la filiale de la Socfin a adopté le nom de Société agricole et forestière africaine (SAFA), Dizangué avait déjà presque 5000 hectares en hévéas. La Socfin a introduit la greffe de boutures et la saignée en spirale pleine de l'Asie du sud-est, et a formé la main-d'œuvre locale à ces techniques $^{2}$. En 1940, Dizangué produisait 1137 tonnes de caoutchouc (Juliat et al., 1951).

Le roi des Belges aurait personnellement persuadé la Socfin de se lancer dans une affaire semblable au Congo belge (Anciaux, 1955). En 1937, la Socfin a envoyé une mission d'études, et a soumis des propositions au gouvernement ${ }^{3}$. Ayant délimité des terres dans la région de Tshuapa, près de Coquilhatville, la Socfin a créé deux nouvelles compagnies, Cultures équatoriales et Compagnie congolaise de l'hévéa (Abeele, 1968 ; Joye et Lewin 1961).

Unilever, firme anglo-néerlandaise, avait une filiale commerciale en Afrique occidentale, la United Africa Company (UAC), issue d'une fusion de compagnies en 1929 (Fieldhouse, 1978). L'UAC a ainsi hérité de quelques plantations d'hévéas au Nigéria et au Cameroun sous tutelle britannique. À partir de 1934, elle a modestement agrandi et amélioré ces plantations, par exemple en plantant 900 hectares d'hévéas greffés à Ikotombo, dans l'est du Nigéria, entre 1934 et 1936. En 1935, la Unilever a transféré ces plantations à une nouvelle filiale, la Pamol, pour laquelle le caoutchouc s'avérait plus rentable que les bananes, l'huile de palme ou le cacao (Fieldhouse, 1994).

\section{4-1939 Les petites plantations}

Des petites plantations de caoutchouc existaient déjà en Afrique, surtout autour de Benin City au Nigéria. Certains villageois Edo dans cette région avaient planté le Funtumia, arbre local, pendant le grand

2. Rapports annuels de la Socfin.

3. Rapport de la Fincol, 1937. 
boom du caoutchouc de la fin du XIX ${ }^{\mathrm{e}}$ siècle, et ils avaient planté des hévéas après 1911 (Igbafe, 1979 ; Hellermann, 2006 ; Buchanan et Pugh, 1955 ; Helleiner, 1966). Même si l'effondrement des prix mondiaux en 1912 a donné un coup d'arrêt à ce processus, on estimait que les Africains disposaient de 2000 hectares d'hévéas au Nigéria en 1926, comparé à 1200 pour les Européens (Whitford et Anthony, 1926).

La remontée du prix mondial dès $1934 \mathrm{a}$ relancé l'enthousiasme, et le Nigéria exportait déjà autour de 2000 tonnes de caoutchouc en 1935. Même si le prix restait assez bas par rapport aux années 1920, les autres produits, surtout le cacao, étaient en plus mauvaise posture (Igbafe, op. cit.). Les anciennes plantations étaient remises en état, et de nouvelles plantations étaient établies. Les chefs et les riches avançaient des fonds aux paysans pauvres leur donnant le droit de planter des hévéas sur leurs terres. Les arbres devenaient ainsi leur propriété, même si la terre elle-même ne l'était pas. Pour mettre fin à cet abus, les Britanniques ont promulgué le Permanent Crops Act en 1937, qui a ralenti ces transferts, tout en contribuant à une fronde grandissante dans la région. En outre, les autorités coloniales se souciaient de disette en 1938, si les Edo continuaient à planter des hévéas à cette cadence (Hellermann, op. cit.).

Ailleurs en Afrique, l'on trouve peu de signes de petites plantations librement établies, mais l'exemple de l'Ouganda montre qu'il faut se méfier des déclarations coloniales. Lord Hailey annonçait en 1938 que tout le caoutchouc était entre les mains des Européens (Hailey, op. cit.). En réalité, les native cultivators avaient aussi planté des hévéas pendant les années 20, en partie pour remplacer le Manihot glaziovii (caoutchouc du Ceará) (Whitford et Anthony, op. cit.). En 1935, à côté des 4800 hectares des Européens, les indigènes cultivaient quelques 400 hectares en hévéas, parfois mélangés avec des caféiers (Mukasa, 1970).
En Afrique équatoriale, la culture du caoutchouc était obligatoire. Au Congo belge, l'hévéa était un des trois produits imposés par le plan agricole de 1933. En pays Mongo, des agronomes européens sélectionnaient les terrains, souvent en bordure d'une nouvelle piste, où les villageois devaient planter 25 à 40 hectares de palmiers à huile et d'hévéas. Les prix de vente, ainsi que les acheteurs, étaient fixés par l'administration. En 1940, presque 2000 hectares étaient ainsi plantés en hévéa dans la région (Nelson, 1994). En Afrique équatoriale française (AEF), le gouvernement a imposé la culture du Manihot, et d'un peu d'hévéa, dans le centresud de l'Oubangui-Chari (République Centrafricaine) (Coquery-Vidrovitch, 1972). Suspendues pendant la grande crise économique, ces plantations ont connu une certaine reprise à partir de 1934 (Kalck, 2005 ; Grande Bretagne, 1942).

\section{9-1945 Les grandes plantations dans la bataille du caoutchouc}

La Seconde Guerre mondiale a rehaussé la valeur stratégique de toutes les matières premières et les exportations africaines de caoutchouc sont passées d'environ 13000 tonnes à plus de 18000 tonnes entre 1939 et 1941 (FAO). Mais le caoutchouc africain n'était qu'un produit d'appoint pendant cette première phase du conflit. Les conventions britanniques avec les gaullistes au Cameroun en octobre 1940, et avec les Belges au Congo en janvier 1941, ne faisaient pas mention de caoutchouc (Joseph, 1977 ; Onana-Mfege, 2006 ; Willame, 1983). Si les accords de mai 1941 entre la Grande Bretagne et les gaullistes en AEF prévoyaient l'achat de tout le caoutchouc exporté, c'était surtout pour soutenir l'économie et les finances de la fédération (Thompson et Adloff, 1960).

Les producteurs africains souffraient aussi de certains effets du conflit. Les sous-marins de l'Axe faisaient des ravages, les Alliés 
manquaient de bateaux et l'appel sous les drapeaux désorganisait les plantations appartenant à des sociétés européennes, en les privant de cadres. Pour éviter l'inflation, le prix du caoutchouc était contrôlé à Londres à partir d'octobre 1941 (Wilson, 1958). En outre, après juin 1940, les Britanniques ont appliqué un blocus aux colonies de l'Italie et de Vichy (Medlicott, 1952-1959). Ils ont aussi exproprié les plantations appartenant aux sujets ennemis, notamment les terres allemandes au Cameroun britannique (Edington, 1991).

La situation s'est transformée de fond en comble entre décembre 1941 et mars 1942, lorsque les neuf dixièmes des hévéas mondiaux furent tombés entre les mains des forces japonaises. Un gros effort fut entrepris par les Alliés pour intensifier la production de caoutchouc synthétique et régénéré, mais il fallait du caoutchouc naturel pour les pneus de grande taille (Allen, 1949). En mars 1943, les Alliés relevèrent le prix fixé à Londres, de 1 shilling et 1,75 pennies à 1 shilling et 6 pennies (Wilson, op. cit.). En 1945, l'Afrique exportait autour de 55000 tonnes, semblable à l'Indochine à la veille de la guerre, et correspondant à $28 \%$ du total mondial de caoutchouc naturel (Bauer, 1948). Le continent s'est aussi regroupé sous la houlette des Alliés, après la chute de l'empire italien et du régime de Vichy.

Comme un hévéa met un minimum de cinq ans pour entrer en production, la solution la plus radicale, à court terme, était la « saignée à mort » (slaughter tapping) des arbres existants. On récupérait des plantations abandonnées, contenant souvent du Manihot ou du Funtumia (India-Rubber Journal, 1942 ; Henry, 1983 ; Bulletin agricole du Congo belge et du Ruanda-Urundi, Bacbru, 1961 ; Serier, 1993). On plantait aussi des hévéas en espacement «ultradense », pour les saigner très jeunes. Cependant, personne ne savait quand la guerre prendrait fin, et l'on craignait que les Japonais ne détruisent les plantations de l'Asie du Sud-Est. L'Afrique a donc bénéficié d'investissements considérables en plantations nouvelles. Comme l'hévéa vit et produit pendant des dizaines d'années, ceci préparait l'avenir du continent.

Dès 1940, la Firestone a intensifié ses méthodes de production au Libéria, répondant au programme de stockage ambitieux des États-Unis. De 5500 tonnes en 1939, la production est passée à 20000 en 1945 , représentant un tiers des exportations du continent. La main-d'œuvre comptait 12500 personnes en 1940, et 29500 en 1947. La compagnie saignait ses arbres plus jeunes que d'habitude, et de manière plus poussée, quoique la Firestone ait évité une politique claire de saignée à mort (Lief, op. cit. ; Taylor, 1956 ; Schulze, 1973 ; Salles, op. cit. ; Serier, op. cit.). En outre, la Firestone construisit une nouvelle usine de latex liquide à Harbel en 1940, devenant la seule source alliée de cette forme de caoutchouc naturel, essentiel pour les gants chirurgicaux et autres usages spécialisés (Taylor, op. cit. ; Lief, op. cit.).

Le groupe Unilever a profité de ses plantations d'hévéas, et en a planté d'autres. Au Nigéria et au Cameroun britannique, le caoutchouc contribuait à hauteur des troisquarts des profits grandissants de la Pamol entre 1941 et 1945 (Fieldhouse, 1994). Une autre filiale, Huileries du Congo belge, a planté du caoutchouc sur deux propriétés en 1942 (Edington, op. cit.).

Après la défaite de la Belgique et de la France, le bureau de la Socfin à Paris tenta de gérer les plantations du groupe en zone Vichy (Clarence-Smith, op. cit.). Robert Michaux, longtemps à la tête des opérations en Malaisie, faisant une tournée d'inspection en Afrique de l'Ouest en 1941, a délimité une zone pour planter le caoutchouc dans le sud-ouest de la Côte d'Ivoire, et lança une station expérimentale de 150 hectares à Bingerville. À partir de janvier 1942, il dirigeait à Paris le nouvel Institut de recherches sur le caoutchouc en Afrique, avec une subvention de Vichy (Padirac, 1993 ; Schoofs, 1944). 
En revanche, Robert Hallet, accompagné de plusieurs cadres belges, rejoignait New York, mettant les plantations de la Socfin au Cameroun et au Congo au service des Alliés, avec l'aide de planteurs rescapés de l'Asie du SudEst (Boulle, 1983). Le «plan Hallet » de 1942 prévoyait la surexploitation des arbres existants au Cameroun, et l'expansion rapide des plantations (Edington, op. cit. ; Serier op. cit.). La Socfin a vendu ses immenses plantations de café en Angola, en 1943-1944, pour financer le «plan dense » au Congo, plantant 2500 hévéas à l'hectare, en vue de les saigner précocement (ASB, 1985). Les grandes plantations adoptèrent des méthodes intensives sur environ 7000 hectares au Congo belge (Becquet, 1945). Pour attirer la main-d'œuvre nécessaire, les patrons ouvraient des cantines et obtenaient des produits importés (Cornelis, 1983).

Les gouvernements coloniaux se sont même faits planteurs. Les terres allemandes au Cameroun britannique, confisquées en septembre 1939, ont d'abord gardé leur personnel. Après l'emprisonnement des Allemands, en juin 1940, les plantations ont temporairement réduit leurs activités, mais la perte de la Malaisie a fortement stimulé le caoutchouc (Epale, 1985 ; Ndi, 1986). Des planteurs fuyant la Malaisie ont fourni leur expertise, et les arbres étaient soumis à des saignées intensives journalières ${ }^{4}$. Au Congo belge, le département de l'Agriculture a repris en main des plantations délaissées de Manihot et de Funtumia (India-Rubber Journal, 4 juillet 1942).

Au Tanganyika, beaucoup de Manihot restaient sur les plantations allemandes délabrées, contrôlées par le Custodian of Enemy Property. Quelques 5500 hectares ont été débroussaillés et saignés par des travailleurs recrutés dans le centre du territoire, et soumis à la discipline militaire, avec l'aide d'un personnel européen réfugié de Malaisie. De 1942 à 1945, l'Afrique du Sud a obtenu autour de 5000 tonnes de ce caoutchouc (Westcott, 1982, 1986).

4. Cf. India-Rubber Journal, 16 janvier 1943.

\section{9-1945 Les petits planteurs et le caoutchouc sauvage}

Les sources tendent à classer comme « sauvage » tout le caoutchouc produit par les Africains pendant la guerre, environ un cinquième des exportations totales, mais il est difficile d'établir une différence claire entre caoutchouc sauvage et cultivé (FAO, op. cit.). En Guinée-Bissau, les nombreuses références aux lianes Landolphia dans la brousse cachaient le fait qu'une partie des exportations venait de petites parcelles de Manihot, cultivées par des Capverdiens immigrés (Monteiro, 1944 ; Fonseca, 1947).

Aussi, quand d'anciennes plantations villageoises étaient remises en condition et saignées, la production paysanne était souvent classée comme étant sauvage, par exemple au Congo belge, en AEF, et au Ghana (Gold Coast) (Becquet, op. cit. ; Weinstein, 1972 ; La Anyane, 1963). Au Congo belge, $7 \%$ des 1127 tonnes exportées en 1939 étaient censées être de cueillette, et $70 \%$ des 7989 tonnes exportées en 1945 (Bacbru, op. cit.). Par contre, l'Afrique occidentale française (AOF) ne produisait officiellement que du caoutchouc sauvage, et l'on ne trouve pas de références à d'anciennes plantations. Les exportations sont passées d'environ 500 tonnes en 1939 à 3000 tonnes par an en 1944-1945, provenant surtout des lianes Landolphia en Guinée-Conakry, et d'arbres Funtumia en Côte d'Ivoire (AOF, 1950, 1951 ; Aubréville, 1949).

On a peut-être exagéré la violence utilisée contre les Africains pour assurer cette cueillette en brousse. La France était la principale puissance accusée, et les gaullistes auraient été encore plus contraignants que les autorités de Vichy (Suret-Canale, 1971 ; Johnson 1972 ; Crubilé, 1950 ; Thompson et Adloff, op. cit. ; Kalck, 1959). Cependant, des témoignages oraux provenant du sudouest de l'Oubangui-Chari présentent une tout autre image. Malgré un souvenir d'abus, les Africains de cette région se lamentent 
plutôt de la mévente du caoutchouc sauvage après la guerre. Le caoutchouc signifiait 1 'accès aux biens de consommation importés, et quand l'Occident n'en voulait plus, ils se trouvaient appauvris (GilesVernick, 2002).

Au Congo belge, l'ordonnance du 10 mars 1942 imposait 60 jours de travail par ans pour certaines tâches, dont la récolte de caoutchouc sauvage (Bacbru, op. cit. ; Henry, op. cit.). Ceci dit, le gouvernement cherchait aussi à offrir un prix rémunérateur, des primes en espèces et en nature, un approvisionnement en biens importés, des moyens de transport, et un encadrement technique (Becquet, op. cit. ; Cornelis, op. cit.)

Quant aux autorités britanniques, elles suivaient surtout une politique des prix ${ }^{5} \mathrm{En}$ Rhodésie du nord (Zambie), le gouvernement a augmenté les prix, tout en appelant à la solidarité dans la guerre (Hobson, 1960 ; information de Achim von Oppen). En Éthiopie, prise aux Italiens en 1941, les Britanniques ont dû payer dix fois le prix officiel pour obtenir la quantité dérisoire de 25 tonnes entre 1943 et 1945 (Hurstfield, 1953). Toutefois, le Wild Rubber Order de 1943 imposait un quota de 3 livres par mois par homme au Cameroun britannique, et un rapport de 1944 se plaignait des méthodes «énergiques » employées par les chefs africains de la région de Bamenda pour faire rentrer le caoutchouc (Epale, op. cit. ; Ndi, op. cit.).

La hausse des prix consentie par les Alliés, qui variait dans différents territoires, a encouragé les paysans africains à planter de l'hévéa, même si les arbres ne pouvaient pas être saignés à temps pour contribuer à l'effort de guerre. Une véritable explosion de la culture du caoutchouc était visible en pays Edo à partir de 1942 (Hellermann, op . cit.). Le gouvernement colonial du Nigéria aidait en reclassant les réserves forestières comme terrains agricoles (Hellermann et Usuanlele, 2009). La Pamol fournissait des graines

5. Cf. India-Rubber Journal (IRJ), 4 juillet 1942. d'hévéas, donnant une forte impulsion à ce processus (Edington, op. cit. ; Imoagene, 1990).

Les gaullistes et les Belges ont aussi soutenu des projets pour accroître ce secteur. Déjà en 1940, Leclerc encourageait la formation de petites parcelles d'hévéa le long des pistes camerounaises (Serier, op. cit.). En 1943, les autorités ont lancé un "plan d'hévéaculture indigène » dans le sud-est du Cameroun, région déshéritée et source principale de caoutchouc sauvage. Le modèle prévoyait des plantations familiales d'un hectare, correspondant aux efforts d'une personne pendant une journée, mais avec la possibilité d'agrandir les exploitations. Les pépinières ont été constituées en 1943, avec des graines fournies par la SAFA, et les premiers plants distribués en 1945 (Juliat et al., op. cit.). Au Gabon, Félix Éboué a suivi une politique semblable dès 1941, créant la station expérimentale d'Oyem pour le « développement de l'hévéaculture indigène » avec des graines de la SAFA, et lançant la culture sur 1200 hectares à Minvoul en 1944-1945 (Crubilé, op. cit. ; Thompson et Adloff, op. cit.). Au Congo belge en 1943, sur environ 50000 hectares d'hévéas, 20000 appartenaient à des indigènes (Becquet, op. cit.).

\section{6-1973 Les grandes plantations}

Les grandes plantations d'hévéas ont continué à se développer en Afrique après la guerre, mais sans opérer la percée sur le marché mondial dont certains rêvaient. Une fois la production repartie en Asie, l'Afrique restait marginale, malgré le repli de certaines sociétés implantées en Indochine et en Afrique française. Le caoutchouc synthétique, bénéficiant de l'abondance de pétrole bon marché et de grands progrès technologiques, faisait des progrès considérables, même si les grands pneus avaient encore besoin de caoutchouc naturel (Morris, 1989 ; Grilli et al., 1980). 
Au Libéria, où la Firestone est restée profondément ancrée, les tentatives de diversifier l'économie vers le cacao, le café, et les bananes ont échoué. Le caoutchouc dominait donc encore l'agriculture de rente, et les exportations croissaient, bien qu' assez lentement. D'autres groupes étrangers ont reçu des concessions, sous le régime de la «porte ouverte », et l'élite Américo-Libérienne a développé ses propres terres, mais la Firestone assurait encore $56,6 \%$ des exportations de caoutchouc en 1969 (Schulze, op. cit.). Toutefois, le minerai de fer a dépassé le caoutchouc dans les exportations en 1961, et l'abolition du travail forcé en 1962, imposée par la communauté internationale, a obligé la Firestone à réduire ses effectifs (Kraaij, op. cit.).

Sous un régime plus souple et pragmatique que ceux de la plupart des pays africains, la Côte d'Ivoire a progressé après 1960, encourageant le caoutchouc pour diversifier l'économie. La Société indochinoise de plantations d'hévéas s'était intéressée au territoire à partir de 1955, et avait créé la Société africaine de plantation d'hévéas (SAPH) en décembre 1956, avec des graines transportées du Vietnam. Avec le groupe Michelin, la SAPH était au devant de la scène après 1960 (Dian, 1985). Partant d'une production modeste de 2000 tonnes en 1965, la Côte d'Ivoire a triplé ses exportations dans les années 1970 (Nigeria 1987 ; Tuinder, 1978). Mais Abidjan a choisi la voie des grandes plantations d'hévéas, au moment même où les petits planteurs triomphaient en Asie (Grilli, 1980).

Au Nigéria, où le gouvernement colonial n'avait jamais beaucoup aidé les grandes plantations, les nationalistes africains se sont montrés plus enthousiastes. Ainsi, les gouvernements régionaux autonomes du sud du Nigéria ont créé leurs propres plantations de caoutchouc dans les années 1950 (Buchanan et Pugh, op. cit.). Malgré un appel à des fonds et à des gérants occidentaux, ces entreprises bureaucratiques fonctionnaient mal. Ensemble, les grandes plan- tations, tant publiques que privées, ne produisaient que $5 \%$ des 60000 tonnes de caoutchouc exportées par le Nigéria en 1961 (Udo, 1965 ; Helleiner, op. cit.). La guerre de Corée a stimulé les profits, mais l'huile de palme rapportait plus que le caoutchouc pour la Pamol à partir de 1947 (Fieldhouse, 1994). D'une manière générale, Unilever s'est repliée sur les activités commerciales de l'UAC, tout en développant un secteur industriel de substitution aux importations (Fieldhouse, 1978). L'UAC s'intéressait donc plutôt aux usines de traitement de latex (Imoagene, op. cit.).

Après l'indépendance en 1960, le premier plan nigérian, couvrant la période 19621968, contenait un programme de grandes exploitations. Malgré une rhétorique antiimpérialiste, ce plan faisait appel aux capitaux étrangers, et leur proposait des avantages considérables. Cependant, les résultats étaient minces, et les résistances locales fortes. Ainsi, la Osse River Rubber Plantation, établie en pays Edo en 1965, a suscité de vives polémiques. La guerre du Biafra (1967-1971) freinait ensuite toute expansion (Hellermann, op. cit.).

$\mathrm{Au}$ Cameroun occidental, les plantations allemandes étaient nationalisées en 1946, pour former la Cameroon Development Corporation (CDC). Les hévéas étaient en assez mauvais état, à la suite de leur surexploitation pendant la guerre, mais la CDC a pris la décision d'évoluer avec cette denrée (Bederman, 1968 ; Buchanan et Pugh, op. cit.). La surface plantée en hévéas a donc plus que doublée entre 1955 et 1975 (Epale, 1985). La Pamol (Unilever) se montrait plus prudente, investissant plutôt dans l'huile de palme que dans le caoutchouc (Courade, 1978).

Pour la Socfin, groupe privé et étranger, cette période a débuté d'une manière houleuse au Cameroun oriental. Aux yeux de la France, l'importance du caoutchouc de Dizangué se trouvait rehaussée par la guerre d'Indochine et en 1954 la SAFA produisait 3100 tonnes. Mais les nationalistes 
camerounais voyaient la SAFA comme une enclave colonialiste et capitaliste. Malgré l'abolition du travail forcé en 1946, les rapports sociaux sur la plantation restaient tendus, et les grèves constantes (Joseph, op. cit.). Néanmoins, un gouvernement remarquablement stable et conservateur après l'indépendance en 1960 a maté l'insurrection de l'Union des populations du Cameroun, et a permis à la Socfin de consolider ses affaires (Suret-Canale, 1972). En 1975, quelques 20000 hectares étaient plantés en hévéas à Dizangué (Edington, op. cit.).

Les grandes plantations n'ont connu qu'un succès modeste en AEF. En 1946, la SAFA (groupe Socfin) a acquis une concession de 3000 hectares dans la forêt de la Lobaye, près de M'Baïki, en OubanguiChari (RAS, 1946 ; Thompson et Adloff, op. cit. ; Kalck, 2005). Plus de 1000 hectares étaient déjà défrichés en 1948 (Crubilé, op. cit.). Mais les Palmeraies et Hévéas du Gabon, filiale d'Unilever fondée en 1956, ont vite mis l'accent sur l'huile de palme (Fieldhouse, 1994).

$\mathrm{Au}$ Congo belge, les patrons plantaient peu après la guerre de Corée, craignant la concurrence du caoutchouc synthétique (Vandewalle, 1966). Néanmoins, les plantations de la Socfin, atteignaient 20000 hectares en 1956 (ASB, op. cit.). Les Huileries du Congo belge (groupe Unilever), avec presque 7000 hectares en hévéas, produisaient plus de 6000 tonnes de caoutchouc en 1959 (Fieldhouse, 1978).

L'indépendance du Congo belge en 1960 se révéla traumatique. Les plantations de la Socfin, étaient finalement «zaïrianisées » en 1974 (ASB, op. cit.). Les Huileries du Congo belge, devenues Plantations Lever au Zaïre, ont réussi à éviter la nationalisation, mais ont souffert de séquelles prolongées d'une décolonisation chaotique. Des milliers d'hectares d'hévéas étaient abandonnés à la brousse dans les années 1980, et les exportations ont chuté (Shapiro et Tollens, 1992).

\section{5-1973 \\ Les petits et moyens planteurs Le Nigéria et les autres}

Les petits planteurs du Nigéria se sont montrés les producteurs les plus dynamiques en Afrique, propulsant leur pays sur le devant de la scène. En 1964, le Nigéria a temporairement dépassé le Libéria comme le plus gros producteur africain de caoutchouc, avec 66000 tonnes. Cette denrée représentait alors entre $6 \%$ et $8,5 \%$ en valeur des exportations totales du Nigéria (Helleiner, op. cit.). La demande locale s'est aussi accrue avec la création par Michelin et Dunlop de deux usines de pneus, en 1962 et 1963 (Kilby, 1975). En 1966, $8 \%$ de la production étaient transformés dans le pays (Nigeria, 1987). Cependant, l'avenir du caoutchouc était déjà menacé, car les producteurs ont cessé de planter des hévéas dans les années 1960. Il semblerait que le prix du caoutchouc, en monnaie locale, n'était plus attirant, comparé à ceux d'autres produits (Imoagene, op. cit.).

Les producteurs Edo ont consolidé leur position de pointe au Nigéria, l'hévéa étant la culture de rente la plus importante de la région (Hellermann, op. cit. ; Nigeria, 1957). Au début des années 1960, $91 \%$ du caoutchouc du Nigéria étaient cultivés sur des parcelles familiales de un à deux hectares (Egboh, 1985). Les hévéas étaient saignés à partir de cinq à sept ans, arrivaient à leur rendement maximal autour de quinze ans et continuaient à produire aux alentours de trente ans (Helleiner, op. cit.). Ces parcelles de caoutchouc étaient aussi plantées en ignames, et autres cultures familiales (Buchanan et Pugh, op. cit.). La plupart des familles disposaient de quelques centaines d'hévéas en 1957, et le caoutchouc était fumé dans des fours sur place (Bradbury et Lloyd, 1957). La qualité et le rendement de ce caoutchouc étaient généralement médiocres (Helleiner, op. cit. ; Imoagene, op. cit. ; Buchanan et Pugh, op. cit.). 
Le boom du caoutchouc était accompagné par une différentiation sociale grandissante. Une paysannerie moyenne exploitait jusqu'à 50 hectares (Helleiner, op. cit.). Certains villageois possédaient des presses pour transformer le caoutchouc en feuilles, les louant à d'autres familles, et devenaient les agents locaux de grosses firmes exportatrices. Quelques planteurs Edo disposaient même de plusieurs centaines d'hectares. Ils habitaient Benin City, obtenaient des titres de chefferie, et se faisaient commerçants en caoutchouc (Bradbury et Lloyd, op. cit.). Un certain Ja-Thomas possédait des usines modernes pour transformer le caoutchouc brut en feuilles (Imoagene, op. cit.). En mars 1953, la Nigerian Rubber Dealers Association voyait le jour à Benin City, manifestant une nouvelle confiance parmi les commerçants africains (Nigeria, 1957).

Une main-d'œuvre abondante et à bon marché était un des atouts des Edo. Ils utilisaient en premier lieu les garçons adolescents de la maisonnée, en ajoutant les femmes de la famille si les prix étaient assez élevés. De plus en plus, ils avaient recours à des immigrés d'ethnie Igbo (Ibo), venant du sud-est du pays, souffrant de surpopulation rurale. Les gros planteurs africains payaient des salaires (Bradbury et Lloyd, op. cit.). Par contre, au niveau du menu peuple, il était plus courant de faire saigner les hévéas par des métayers ou des fermiers (Hellermann, op. cit.).

Les espoirs suscités par la Seconde Guerre mondiale n'ont guère été suivis d'effets ailleurs qu'en Afrique de l'Ouest. Les petites et les moyennes plantations africaines ont bien connu un certain progrès après 1945 au Libéria (Schulze, op. cit. ; Salles, op. cit.). Mais ces nouveaux planteurs étaient surtout des absentéistes vivant en ville, des employés du gouvernement soutenus par la Firestone pour des raisons plus politiques qu'économiques (Kraaij, op. cit.). Au Cameroun occidental, les Bakwiri, population autochtone de la région du Mont Cameroun, n'ont pas réussi à se faire restituer leurs terres par la CDC, même si cette dernière s'est trouvée obligée d'intégrer des petits planteurs de caoutchouc dans ses opérations à partir de 1964 (Epale, op. cit. ; Bederman, op. cit.). Au Cameroun oriental, seulement 300 tonnes sur les 3100 exportées provenaient de producteurs autres que la SAFA en 1954, les plans pour le sud-est ayant échoué (Joseph, op. cit.). Même en Côte d'Ivoire, sous un président ancien planteur, les petits producteurs ne représentaient que $4 \%$ de la production en 1979 (Dian, op. cit.).

La situation était encore plus décourageante en Afrique équatoriale, où les autorités françaises et belges avaient longtemps obligé les paysans à planter des hévéas. Cet état de fait aurait contribué au manque de succès du programme lancé par Félix Éboué au Gabon (Crubilé, op. cit.). Au Congo belge, les petits planteurs disposaient de 28000 hectares d'hévéas, à peu près $40 \%$ de la surface plantée à la fin de la guerre (Bacbru, op. cit. ; Becquet, op. cit.). Le gouvernement colonial restait optimiste en 1950, estimant que les plantations indigènes « entrent graduellement en production » (Belgique, 1951). Mais en 1958 les indigènes ne produisaient que 3576 tonnes, sur un total de 37422 (Bacbru, op. cit.).

\section{Conclusion}

Les conditions qui avaient favorisé l'expansion de l'hévéa en Afrique à partir des années 1930 se sont inversées quarante ans plus tard. Une bonne partie de l'Asie était sortie de ses crises. La Malaisie avait maté l'insurrection communiste autour de 1960, et la situation en Indonésie s'était transformée après la chute de Sukarno en 1965. La mainmise communiste sur les plantations indochinoises, rendue définitive en 1975 , était compensée par un boom en Thaïlande (Baulkwill, 1989 ; Barlow et al., 1994).

Enfin et surtout, en dépit de quelques exceptions, les indépendances en Afrique, à partir de 1957, ont généré des politiques et 
des pratiques économiques désastreuses. En dehors de la zone CFA, les monnaies étaient surévaluées. Les caisses étatiques remplaçaient les commerçants, et offraient des prix dérisoires aux petits producteurs. $\mathrm{La}$ corruption s'étendait de plus en plus. Les grandes plantations privées ont sombré dans les troubles politiques et sociaux qui ont déchiré le Congo après 1960, et leurs fiefs libériens et camerounais étaient menacés par des problèmes semblables. Les plantations étatiques recevaient bien de l'aide des gouvernements, mais elles étaient minées par la mauvaise gestion.

Quant aux petits planteurs du Nigéria, ils faisaient face aux affres d'une économie dominée par le pétrole après 1973 (Hellermann, op. cit.). La production nationale de caoutchouc stagnait autour de 60000 tonnes par an dans les années 1970, et baissait audessous de 50000 tonnes au début des années 1980, laissant de nouveau la première place en Afrique au Libéria. Le taux de change et la politique des prix pratiqués par le gouvernement décourageaient les planteurs (Nigeria, 1987). En outre, la désorganisation des transports et du commerce rendait rares et chères les importations essentielles. Les rendements des hévéas plantés dans les années 1940 et 1950 baissaient, et les paysans ne renouvelaient plus leurs stocks d'arbres (Imoagene, op. cit.). Les producteurs Edo subissaient aussi les conséquences du manque de dragage des bouches du Niger, qui les obligeait à envoyer leur caoutchouc par voie de terre au port engorgé de Lagos ${ }^{6}$. Contrairement au régime Suharto en Indonésie, le gouvernement nigérian n'a pas su exploiter la manne pétrolière pour stimuler le secteur agricole.

La concurrence du caoutchouc synthétique s'est nettement amoindrie à partir de 1973. La hausse brutale des prix du pétrole a augmenté les coûts du synthétique, et la diffusion des pneus à carcasse radiale nécessite plus de caoutchouc naturel (Grilli, op. cit. ; Baulkwill, op. cit.). Or, la Côte d'Ivoire fut le seul producteur africain à saisir cette occasion (Dian, op. cit. ; Onyemelukwe 1984). Ce n'est que pendant les décades récentes que de nouvelles possibilités pour l'hévéa se sont développées en Afrique, du fait, surtout, du dynamisme de l'agriculture familiale (Ruf, 2009 ; Mesike et Ubani, 2008).

6. $C f$. AllAfrica.com, 2009. 


\section{RÉFÉRENCES BIBLIOGRAPHIQUES}

Abeele M. van den (1968). Hallet Robert. In «Biographie belge d'outremer", Bruxelles, Académie Royale des sciences d'Outre-Mer, VI, 450-2.

AOF (1950). Annuaire statistique de l'Afrique occidentale française, édition 1949. Paris, Imprimerie Nationale.

AOF (1951). Inspection générale de l'agriculture. Rapport annuel 1950.

AllAfrica.com (2009). Nigeria: FG killed Delta ports, says Iweta. 5 octobre, http://allafrica.com/stories/printable/200910050794.html

Allen H. (1949). The house of Goodyear: fifty years of men and industry. Cleveland $(\mathrm{OH})$ : [Goodyear].

Anciaux L. (1955) La participation des Belges à l'œuvre coloniale des Hollandais aux Indes orientales. Bruxelles, Institut Royal Colonial Belge.

ASB (1985). Robert Hallet. Veldekens F, Bruxelles, Archives de la Socfin.

Aubréville A. (1949). Les forêts. In Eugène Guernier, (éd.) "Afrique occidentale française », Paris, Encyclopédie coloniale et maritime, vol. 1, p. 371-90.

BACBRU (1961). Bulletin agricole du Congo Belge et du Ruanda-Urundi. Volume jubilaire, 1910-1960, Bruxelles, Ministère du Congo Belge et du RuandaUrundi.

Barlow C., Drabble J. (1990). Government and the emerging rubber industries in Indonesia and Malaya, 1900-1940. In Booth Anne, O’Malley W.-J., Weidemann A., (eds.), «Indonesian economic history in the Dutch colonial era », New Haven, Yale University Press, p. 187-209.

Barlow C., Jayasuriya S., Tan Suan C. (1994). The world rubber industry. Londres, Routledge.

Bauer P.-T. (1948). The rubber industry, a study in competition and monopoly. London, Longmans Green \& Co.
Baulkwill W.-J. (1989). The history of natural rubber production. In Webster C.-C., Baulkwill W.-J. (eds.), « Rubber», Harlow, Longman, p. 1-56.

Becquet A. (c. 1945). Le caoutchouc. In «Congo belge 1944 », Léopoldville, Imprimerie du Gouvernement général, p. 72-3.

Bedermann S.-H. (1968). The Cameroons Development Corporation, partner in national growth. Bota, Cameroons Development Corporation.

Belgique (1951). Rapport sur l'Administration de la colonie du Congo Belge pendant l'année 1950, présenté aux chambres legislatives. Bruxelles, Edimco.

Boulle P. (1983). Sacrilege in Malaya. Kuala Lumpur, Oxford University Press. Bradbury R.-E., Lloyd P.-C. (1957). The Benin kingdom and the Edo-speaking peoples of south-western Nigeria, together with a section on the Itsekiri. Londres, International African Institute.

Buchanan K.-M., Pugh J.-C. (1955). Land and people in Nigeria: the human geography of Nigeria and the environmental background. London, University of London Press.

Clarence-Smith W.-G. (1997). The RivaudHallet plantation group in the economic crises of the inter-war years. In Lanthier P., Watelet H. (eds.), «Private enterprises during economic crises: tactics and strategies », Ottawa, Legas, p. 117-32.

Clarence-Smith W.-G. (2010). La SOCFIN (Groupe Rivaud) entre l'Axe et les Alliés. In Bonin H., Bouneau C., Joly H. (eds.), «Les enterprises et l'outre-mer français pendant la Seconde Guerre mondiale », Pessac, Maison des Sciences de l'Homme d'Aquitaine, p. 99-113.

Coquery-Vidrovitch C. (1972). Le Congo au temps des grandes compagnies concessionnaires, 1898-1930. Paris, Mouton. 
Cornelis H.-A.-A. (1983). Belgisch Congo en Ruanda-Urundi tijdens de Tweede Wereldoorlog: de economische en financiële situatie. In «Le Congo Belge durant la Seconde Guerre mondiale », Bruxelles, Académie Royale des Sciences d'OutreMer, p. 51-81.

Courade G. (1978). Les plantations industrielles d'Unilever au Cameroun (plantations Pamol du Cameroun Limited). Cahiers de l'ONAREST, vol. 1, $\mathrm{n}^{\circ} 2$, p. 91-159.

Crubilé D. (1950). Le caoutchouc. In Guernier E. (éd.), «Afrique-Équatoriale française », Paris, Encyclopédie coloniale et maritime, p. 317-18.

Dian B. (1985). L'économie de plantation en Côte d'Ivoire forestière. Abidjan, Les Nouvelles Éditions Africaines.

Drabble John H. (1973). Rubber in Malaya, 1876-1922: the genesis of the industry. Kuala Lumpur, Oxford University Press.

Edington J.-A.-S. (1991). Rubber in West Africa. Londres, Rex Collings.

Egboh E.-O. (1985) Forestry policy in Nigeria, 1897-1960. Nsukka, University of Nigeria Press.

Epale S. J. (1985) Plantations and development in Western Cameroon, 1885 1975. New York, Vantage Press.

Etoga Eily F. (1971). Sur les chemins du développement, essai d'histoire des faits économiques du Cameroun. Yaoundé, Centre d'Édition et de Production de Manuels et d'Auxiliaires de l'Enseignement.

FAO (Food and Agriculture Organization) (1947). Yearbook of food and agricultural statistics. Washington.

Fieldhouse D.-K. (1978). Unilever overseas, the anatomy of a multinational, 1895-1965. Londres, Croom Helm.

Fieldhouse D.-K. (1994). Merchant capital and economic decolonization: the United Africa Company, 1929-1987. Oxford, Clarendon Press.

Fonseca A.-X. da (1947). A produção de borracha no nosso império e o consumo nacional. Boletim Geral das Colónias, vol. $23, \mathrm{n}^{\circ} 268$, p. 51-5.

Giles-Vernick T. (2002). Cutting the vines of the past: environmental histories of the Central African rain forest. Charlottesville, University Press of Virginia. Grande Bretagne, Naval Intelligence Division (1942). French Equatorial Africa and Cameroons. Oxford, HMSO.

Grilli E.-R., Agostini B.-B., t HooftWelvaars M. J. (eds.) (1980). The world rubber economy: structure, changes and prospects. Baltimore, The Johns Hopkins University Press (World Bank Staff Occasional Papers).

Hailey W.-M. (1938). An African survey: a study of problems arising in Africa south of the Sahara. Londres, Oxford University Press.

Helleiner G.-K. (1966). Peasant agriculture, government, and economic growth in Nigeria. Homewood, Illinois, Richard D. Irwin.

Hellermann P. Von (2006). Things fall apart? A political ecology of twentiethcentury forest management in Edo state, southern Nigeria. PhD Thesis, University of Sussex.

Hellermann P. Von, Usuanlele U. (2009). The owner of the land: the Benin obas and colonial forest reservation in the Benin Division, southern Nigeria. Journal of African History, $\mathrm{n}^{\circ}$ 50, p. 233-46.

Henry J.-M. (1983). L'INEAC en Afrique pendant la Seconde Guerre mondiale. In «Le Congo Belge durant la deuxième guerre mondiale », p. 313-97, Bruxelles, Académie Royale des Sciences d'OutreMer.

Hobson R.-H. (1960). Rubber: a footnote to Northern Rhodesian history. Livingstone, Rhodes-Livingstone Museum.

Hurstfield J. (1953). The control of raw materials, Londres, HMSO.

Igbafe P.-A. (1979). Benin under British administration: the impact of colonial rule on an African kingdom, 1897-1938. Londres, Longman. 
Imoagene O. (1990). The Edo and their neighbours of Midwestern Nigeria. Agodi, New-Era Publishers.

Johnson R.-W. (1972). French imperialism in Guinea. In Owen R., Sutcliffe B. (eds.), "Studies in the theory of imperialism », Londres, Longman, p. 230-47.

Joseph R. (1977). Radical nationalism in Cameroon. Oxford, Clarendon Press.

Joye P., Lewin R. (1961). Les trusts au Congo. Bruxelels, Société Populaire d'Éditions.

Juliat R. et al. (1951). Le Cameroun, pays agricole. In Guernier E., Briat R. (eds.), «Cameroun ; Togo », Paris, Encyclopédie coloniale et maritime, p. 173-227.

Kalck P. (1959). Réalités oubanguiennes. Paris, Berger-Levrault.

Kalck P. (2005). Historical dictionary of the Central African Republic. Lanham (MD), Scarecrow Press.

Kilby P. (1975). Manufacturing in colonial Africa. In Duignan P., Gann L.-H. (eds.), "Colonialism in Africa », vol. 4, the economics of colonialism, Cambridge, Cambridge University Press, p. 470-520. Kraaij F.-P.-M. Van der (1983). The open door policy of Liberia: an economic history of modern Liberia. Bremen, Übersee-Museum.

La Anyane S. (1963). Ghana agriculture: its economic development from early times to the middle of the twentieth century. Londres, Oxford University Press.

Lief A. (1951). The Firestone story: a history of the Firestone Tire and Rubber Company. New York, McGraw-Hill.

McFadyean A. (1944). The history of rubber regulation, 1934-1943. Londres, George Allen and Unwin.

Medlicott W.-N. (1952-1959). The economic blockade. Londres, HMSO.

Mesike C.-S., Ubani S.-E. (2008). Resourceuse efficiency among smallholders in Edo and Delta states of Nigeria: implications on output and environmental degradation. Journal of Agricultural and Social Sciences, $\mathrm{n}^{\circ} 4$, p. 180-2.
Mitchell B.-R. (2007). International historical statistics: Africa, Asia and Oceania, 1750-2005. Basingstoke, Palgrave Macmillan.

Monteiro R. Vaz (1944). Relatório do governador. Bissau.

Morris P.-J.-T. (1989). The American synthetic rubber research program. Philadelphia, University of Pennsylvania Press.

Mukasa S.-K. (1970). Rubber. In Jameson J.-D. (ed.), "Agriculture in Uganda », Londres, Oxford University Press, p. 210-11.

Ndi A. (1986). The Second World War in southern Cameroon and its impact on mission-state relations. In Killingray D., Rathbone R. (eds.), "Africa and the Second World War», Basingstoke, Macmillan, p. 204-31.

Nelson S.-H. (1994). Colonialism in the Congo basin, 1880-1940. Athens, Ohio University.

Nigeria (1957). Handbook of commerce and industry in Nigeria. Lagos, Federal Department of Commerce and Industries.

Nigeria (1987). Reports on the proceedings of the national conference on rubber, wood, and wood products held in Benin City on $16^{\text {th }}$ and $17^{\text {th }}$ February 1987. Lagos, Export Promotion Council.

Onana-Mfege A.-H. (2006). Les Camerounais et le Général de Gaulle. Paris, L'Harmattan.

Onyemelukwe Joe O.-C. (1984). Industrialisation in West Africa. London, Croom Helm.

Padirac R. de (1993). L'Institut de Recherches sur le Caoutchouc, 19361984. Montpellier, CIRAD.

RAS (1946). Rapport Annuel de la Socfin. Ruf F. (2009). L'adoption de l'hévéaculture en Côte d'Ivoire : prix, imitation, et changement écologique. $3^{\mathrm{e}}$ journées de recherches en sciences sociales, Montpellier, 9-11 décembre.

Salles P. (1979). Plantations au Libéria. In Cauvin C., Cordonnier J.-C., Martin M. A., et Salles, P. «Plantations industrielles 
et productions paysannes (Côte d'Ivoire, Libéria, Cambodge, Indonésie) », Talence, Centre d'Études de Géographie Tropicale, p. 158-232

Schulze W. (1973). Liberia: länderkundliche Dominanten und regionale Strukturen, Darmstadt, Wissenschaftliche Buchgesellschaft.

Schoofs M. (1944). La préparation du caoutchouc en extrême-orient. Bruxelles, Ministère des Colonies.

Serier J.-B. (1993). Histoire du caoutchouc. Paris, Desjonquères.

Shapiro D., Tollens E. (1992). The agricultural development of Zaire, Aldershot, Avebury.

Suret-Canale J. (1971). French colonialism in tropical Africa, 1900-1945. Londres, Hurst C.

Suret-Canale J. (1972). Afrique noire, occidentale et centrale. Tome 3, de la colonisation aux indépendances, Paris, Éditions Sociales.

Taylor W.-C. (1956). The Firestone operations in Liberia. Washington, National Planning Association.

Thompson V., Adloff R. (1960). The emerging states of French Equatorial Africa, Stanford, Stanford University Press.

Tuinder B. den (1978). Ivory coast: the challenge of success. Baltimore, Johns Hopkins University Press.
Udo R.-K. (1965). Sixty years of plantation agriculture in southern Nigeria. Economic Geography, vol. 41, n ${ }^{\circ}$, p. 356-68.

Vandewalle G. (1966). De conjuncturele evolutie in Kongo en Ruanda-Urundi, van 1920 tot 1939, en van 1949 tot 1958 . Gent, Rijksuniversiteit te Gent.

Weinstein B. (1972). Éboué. Londres, Oxford University Press.

Westcott N. (1982). The impact of the Second World War on Tanganyika, 19391951. $\mathrm{PhD}$ thesis, Cambridge University.

Westcott N. (1986). The impact of the Second World War on Tanganyika, 19391949. In Killingray D., Rathbone R. (eds.), «Africa and the Second World War, 143-59 », Basingstoke, Macmillan.

Whitford H.-N., Anthony A. (1926). Rubber production in Africa. Washington, US Department of Commerce and Trade, Promotion Series, $\mathrm{n}^{\circ} 34$.

Willame J.-C. (1983). Le Congo dans la guerre : la coopération économique belgoalliée de 1940 à 1944. In "Le Congo belge durant la deuxième guerre mondiale », Bruxelles, Académie Royale des Sciences d'Outre-Mer, p. 213-52.

Wilson J. (1958). The Singapore rubber market. Singapore, Eastern Universities Press. 


\section{ANNEXES}

Tableau 1. Production mondiale de caoutchouc naturel moyenne annuelle (1930-1979) (En milliers de tonnes)

\begin{tabular}{c|c|c|c} 
& Total & Afrique & Amérique latine \\
\hline $1930-1939$ & 904 & 7 & 14 \\
\hline $1940-1949$ & 986 & 39 & 36 \\
\hline $1950-1959$ & 1908 & 97 & 26 \\
\hline $1960-1969$ & 2447 & 162 & 31 \\
\hline $1970-1979$ & 3475 & 209 & 36 \\
\hline \multicolumn{4}{|c}{ Source : Barlow et al. (1994), tableau A3 }
\end{tabular}

Tableau 2. Production mondiale de caoutchouc synthétique moyenne annuelle (1930-1989) (En milliers de tonnes)

\begin{tabular}{r|r}
\hline $\begin{array}{c}\text { En milliers } \\
\text { de tonnes }\end{array}$ & Total \\
\hline $1930-1939$ & 28 \\
\hline $1940-1949$ & 569 \\
\hline $1950-1959$ & 1447 \\
\hline $1960-1969$ & 3721 \\
\hline $1970-1979$ & 7564 \\
\hline $1980-1989$ & 9069 \\
\hline
\end{tabular}

Source : Barlow et al. (1994), tableau A4

Tableau 3. Production de caoutchouc naturel par les principaux pays producteurs africains, moyenne annuelle (1930-1979) (En milliers de tonnes)

\begin{tabular}{c|c|c|c|c|c}
\hline $\begin{array}{c}\text { En milliers } \\
\text { de tonnes }\end{array}$ & Libéria & Côte d'Ivoire & Nigéria & $\begin{array}{c}\text { Cameroun } \\
\text { (est et ouest) }\end{array}$ & $\begin{array}{c}\text { Congo } \\
\text { (Kinshasa) }\end{array}$ \\
\hline $1930-1939$ & 1 & - & 2 & 3 & 1 \\
\hline $1940-1949$ & 18 & - & 7 & 5 & 5 \\
\hline $1950-1959$ & 38 & - & 31 & 5 & 25 \\
\hline $1960-1969$ & 51 & 3 & 62 & 11 & 33 \\
\hline $1970-1979$ & 79 & 15 & 60 & 16 & 32 \\
\hline
\end{tabular}

Sources : Mitchell (2007) ; Nigeria (1987) ; FAO ; McFadyean (1944), tableau 3 